\title{
Recent STAR heavy-ion results
}

\author{
Mikhail Tokarev ${ }^{1, \star}$ (for the STAR Collaboration)
}

${ }^{1}$ Joint Institute for Nuclear Research, 141980 Dubna, Russia

\begin{abstract}
The recent STAR heavy-ion results obtained in the first phase of the RHIC Beam Energy Scan program are presented. The measurements of particle spectra have been performed over a wide range of collision energy $\sqrt{s_{N N}}=7.7-200 \mathrm{GeV}$, centrality and transverse momentum of produced particles. The fixed target mode in heavy-ion collisions at the STAR experiment also extends considerably the range of search for the new physics. Heavy quarks provide an exceptional probe in understanding properties of the hot and dense medium created in such collisions. The Heavy Flavor Tracker (HFT) and Muon Telescope Detector (MTD) upgrades at the STAR experiment at RHIC significantly improved the experimental capabilities of TPC, ToF and EMC detectors in measuring both open and hidden heavy flavor hadrons in heavy-ion collisions.
\end{abstract}

\section{Introduction}

The main goal of the physics program at the Relativistic Heavy Ion Collider (RHIC) is to produce a new state of the nuclear matter in collisions of heavy ions and to investigate its properties over a wide range of energy, centrality with various probes and to determine the features of the phase diagram of the nuclear matter [1-4]. Experimental results from the RHIC support the hypothesis that a stronglycoupled nuclear medium with partonic degrees of freedom, namely the Quark-Gluon Plasma (QGP), is created in heavy-ion collisions at high energy. It is assumed that the medium produced in heavy-ion collisions is thermalized. Therefore the phase diagram is usually presented in the temperature-baryon chemical potential plane $\left\{T, \mu_{B}\right\}$. Both quantities can be varied by changing the energy and centrality of collision and the type and momentum of produced particle. The phase diagram should demonstrate a possible transition from a high energy density and high temperature phase in which quark and gluon degrees of freedom dominate to a phase where the relevant degrees of freedom are hadronic [5-9]. The new state at the top RHIC energy is found to be characterized by the suppression of the high transverse momentum $\left(p_{T}\right)$ hadron production in $\mathrm{Au}+\mathrm{Au}$ collisions relative to $p+p$ collisions [10], large elliptic flow $\left(v_{2}\right)$ for hadrons with light and heavier strange valence quarks, differences between baryon and meson $v_{2}$ at intermediate $p_{T}$ for $\mathrm{Au}+\mathrm{Au}$ collisions [11,12], enhanced correlated yields at large $\Delta \eta$ and $\Delta \phi \simeq 0$ [13]. These properties are associated with the existence of a quark-gluon phase of the nuclear matter.

The description of the experimentally found phase diagram of the nuclear matter in the framework of the Quantum Chromodynamics (QCD) is one of the important problems for the theory. It is the best

\footnotetext{
^e-mail: tokarev@jinr.ru
} 
test of the QCD predictive force especially in the nonperturbative region. Lattice QCD calculations (see [14] and references therein) indicate that the system produced at $\mu_{B}=0$ evolves through a rapid crossover in the quark-hadron phase transition. For collisions corresponding to large $\mu_{B}$, the transition is expected to be first order. The point in the $\left\{T, \mu_{B}\right\}$ plane where the first order phase transition ends, is the QCD critical point. Searching for correspondence between the features (critical point, phase boundaries, type of phase transition) of QCD and experimentally found phase diagram of the nuclear matter is the current focus of the experimental and theory investigations [15]. Therefore the Beam Energy Scan (BES) Program planned at RHIC is aimed to clarify these problems [5-9]. The guiding idea is to vary the collision energy and look for the signatures of phase boundary and critical point i.e. to span the phase diagram from the top RHIC energy (lower $\mu_{B}$ ) to the lowest possible energy (higher $\mu_{B}$ ). We assume that the signatures of QGP created at $\sqrt{s_{N N}}=200 \mathrm{GeV}$ are established [14]. Therefore turn-off of these signatures at particular energy would suggest the crossing of the phase boundary. Similarly, near critical point, there would be enhanced fluctuations in multiplicity distributions of conserved quantities (net-charge, net-baryon) and correlations in distributions of correlation functions (HBT, forward-backward multiplicity). Large fluctuations or divergence of some observables at fixed kinematic and dynamical conditions is usually considered as a characteristic behavior of the system near a critical point. Before looking for these signatures, one should know the region $\left(\left\{T, \mu_{B}\right\}\right)$ and mean transverse momentum $\left(<p_{T}>\right)$ of the phase diagram which can be accessed.

The thermodynamic parameters $T$ and $\mu_{B}$ can be obtained from the ratios and spectra of the produced particles assuming that the thermodynamical equilibrium of the produced nuclear system has been reached. The properties of bulk medium such as the multiplicity density, mean $p_{T}$ may provide insight about the particle production mechanisms at these energies. The systematic study of these bulk properties with light and heavy probes may clarify the evolution and change in behavior of the system formed in heavy-ion collisions as a function of collision energy and centrality.

We will present the recent STAR heavy-ion results obtained in the first phase of the RHIC Beam Energy Scan program. The measurements of particle spectra have been performed over a wide range of collision energy $\sqrt{s_{N N}}=7.7-200 \mathrm{GeV}$, centrality and transverse momentum of produced particles. The fixed target mode in heavy-ion collisions at the STAR experiment also extends considerably the range of search for the new physics. Heavy quarks $(D, J / \psi)$ provide an exceptional probe in understanding properties of the hot and dense medium created in such collisions. The Heavy Flavor Tracker (HFT) and Muon Telescope Detector (MTD) upgrades at the STAR experiment at RHIC significantly improved the experimental capabilities of TPC, ToF and EMC detectors in measuring both open and hidden heavy flavor hadrons in heavy-ion collisions.

\section{STAR detector}

The main detectors of the STAR experiment used to obtain the results on $p_{T}$ spectra, yields, and particle ratios for charged hadrons are the Time Projection Chamber (TPC) [18] and Time-Of- Flight detectors (ToF) [19]. The TPC is the primary tracking device at STAR. It is $4.2 \mathrm{~m}$ long and $4 \mathrm{~m}$ in diameter. Its uniform acceptance covers about \pm 1 units of pseudorapidity $(\eta)$ and the full azimuthal angle. TPC is placed in the uniform solenoidal magnetic field $(0.5 \mathrm{~T})$. The sensitive volume of the TPC contains $\mathrm{P} 10$ gas (10\% methane, $90 \%$ argon) regulated at 2 mbar above atmospheric pressure. The TPC data are used to determine particle trajectories, momenta, and particle-type through ionization energy loss $(d E / d x)$. The TOF is based on Multi-gap Resistive Plate Chamber (MRPC) technology and is very useful to identify the particles at relatively higher momentum range compared to TPC. The Multi-Gap Resistive Plate Chamber Time of Flight (MRPC ToF) [20] will completely surround the outer radius of the TPC, $|\eta|<0.9,0<\phi<2 \pi$. It consists of $23 \mathrm{~K}$ channels from 120 modules. The details of the design and other characteristics of the STAR detectors can be found in [17]. 


\section{Beam Energy Scan at STAR}

The Beam Energy Scan program at RHIC is aimed to perform systematic study and data analysis of the particle production in $\mathrm{Au}+\mathrm{Au}$ collisions over a range of collisions energy $\sqrt{s_{N N}}=7.7-39 \mathrm{GeV}$ and centrality [5-9]. It concerns as spectra of non- and identified particles, ratio of particle yields etc.

\subsection{Nuclear modification factor}

The nuclear modification factor, $R_{C P}$, is the binary collisions $N_{\text {bin }}$ scaled ratio of central $(C)$ to peripheral $(P)$ transverse momentum distributions

$$
R_{C P}=\frac{\left(N_{b i n}\right)_{P}}{\left(N_{b i n}\right)_{C}} \frac{\left(d^{2} N_{A} / d p_{T} d \eta\right)_{C}}{\left(d^{2} N_{A} / d p_{T} d \eta\right)_{P}}
$$

If hard processes were not affected by the presence of the medium, they should scale with the number of binary collisions and $R_{C P}$ would equal unity. All high $p_{T}$ particle species as shown in [21] are strongly suppressed by a factor of 5 in the central $\mathrm{Au}+\mathrm{Au}$ collisions at $\sqrt{s_{N N}}=200 \mathrm{GeV}$. The suppression at top RHIC energy provides evidence for partonic energy loss in QGP.

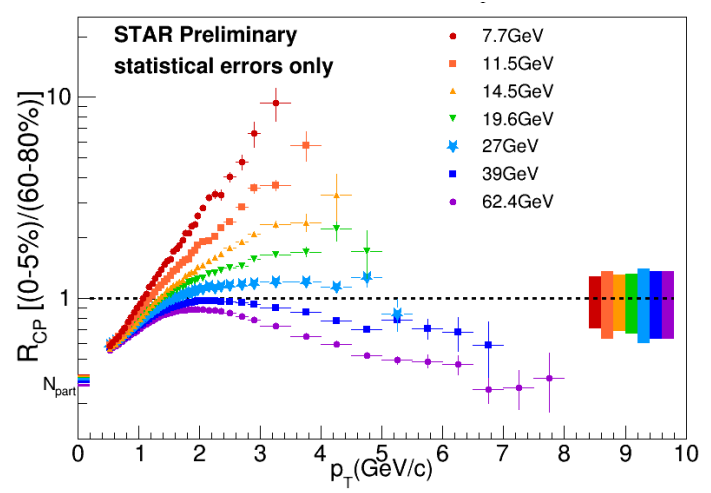

Figure 1. The nuclear modification factor $R_{C P}$ : the ratio of the charged particle invariant yields in $\mathrm{Au}+\mathrm{Au}$ collisions at the energy $\sqrt{s_{N N}}=7.7-62.4 \mathrm{GeV}$ in the range $|\eta|<0.5$ for the centrality $(0-5) \%$ and $(60-80) \%$ versus transverse momentum $p_{T}[22,23]$.

Figure 1 shows the dependence of the ratio of the charged particle invariant yields in $\mathrm{Au}+\mathrm{Au}$ collisions at the energy $\sqrt{s_{N N}}=7.7,11.5,14.5,19.6,27,39$, and $62.4 \mathrm{GeV}$ in the range $|\eta|<0.5$ for the centrality (0-5)\% and (60-80)\% versus transverse momentum $p_{T}$ [22, 23]. The ratio $R_{C P}$ demonstrates strong enhancement of high- $p_{T}$ charged hadrons for the lower collision energies in several ranges of $p_{T}$. For high $p_{T}$, a smooth transition from suppression to enhancement is observed with decreasing $\sqrt{s_{N N}}$.

\subsection{Hadron spectra in $\mathrm{Au}+\mathrm{Au}$}

The transverse momentum spectra of hadrons produced in high-energy collisions of heavy ions reflect features of constituent interactions in the nuclear medium. The medium modification is one of the 
effects (recombination, coalescence, energy loss, multiple scattering,...) that affects the shape of the spectrum. The properties of the created medium are experimentally studied by variation of the event centrality and collision energy. The particle spectra were found to reveal the exponential and power behavior at low- and high- $p_{T}$ region, respectively.

Figure 2 shows the transverse momentum spectra of $\pi^{+}, K^{+}, p, \Xi^{-}, \Lambda$ and $\bar{p}$ particles produced in $\mathrm{Au}+\mathrm{Au}$ collisions at $\sqrt{s_{N N}}=14.5 \mathrm{GeV}$ for different centralities and in the central rapidity range [24]. Errors shown are statistical only. The different fit functions shown by the dashed lines were used to obtain yields. These are Bose-Einstein function for $\pi^{+},\left(m_{T}-m\right)$ exponential function for $K^{+}$, double exponential function for $p, \bar{p}$ and and Boltzmann function for $\Xi^{-}, \Lambda$. The spectra are measured using TPC+TOF up to $4 \mathrm{GeV} / \mathrm{c}$ for strange baryons and less than $2 \mathrm{GeV} / \mathrm{c}$ for $\pi, K, p, \bar{p}$. The similar spectra for identified particles at other BES energies were presented in [25-27].
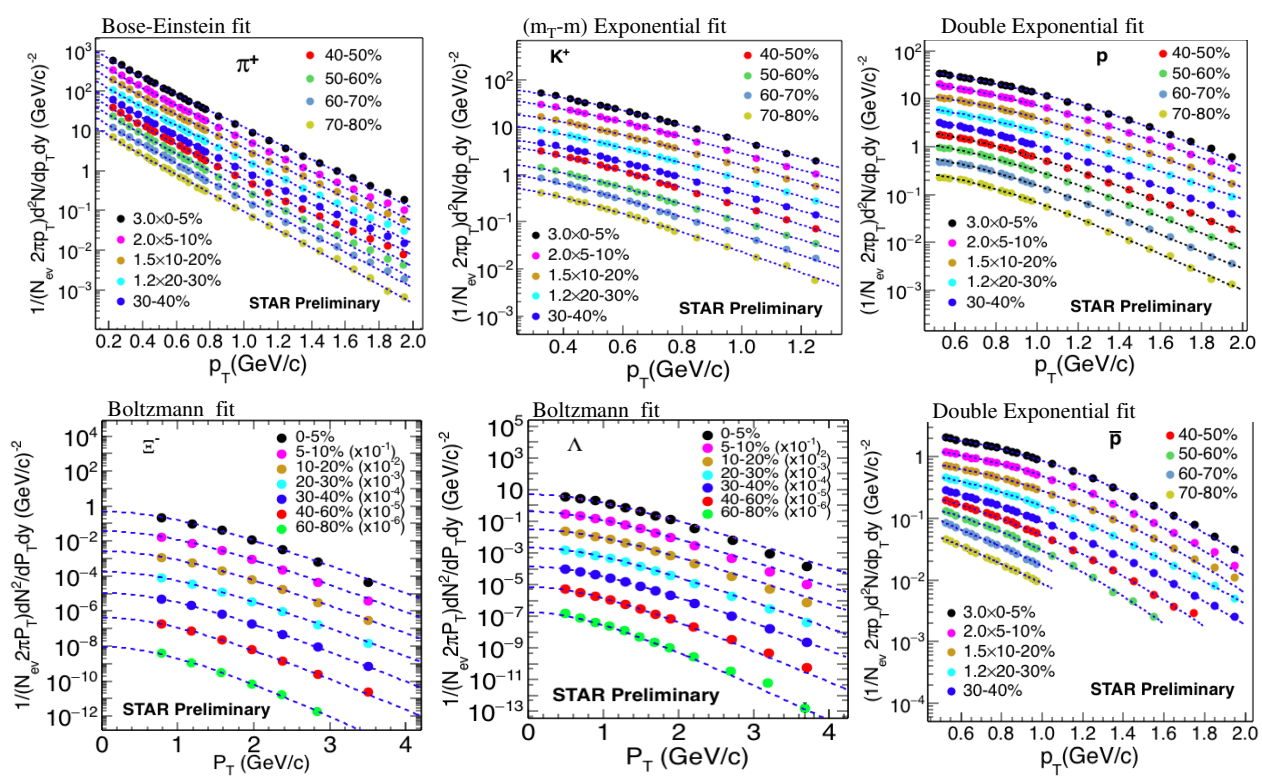

Figure 2. Mid-rapidity $(|y|<0.5)$ transverse momentum spectra for $\pi^{+}, K^{+}, p, \Xi^{-}, \Lambda$ and $\bar{p}$ in Au+Au collisions at $\sqrt{s_{N N}}=14.5 \mathrm{GeV}$ for different centralities. The curves represent Bose-Einstein function, $\left(m_{T}-m\right)$-exponential, Boltzmann and double-exponential function fits. The errors are statistical [24].

Multi-strange hadrons are important probes for the search of the phase boundaries of QGP (see [28] and references therein). The $\Omega$ hyperons and $\phi$ mesons are expected to have relatively small hadronic interaction cross sections. Therefore, they can carry the information directly from the chemical freeze-out stage with little or no distortion due to hadronic rescattering. Yields of these particles are minimally distorted from decay feed-down also. Therefore $\Omega$ hyperons and $\phi$ mesons offer a unique advantage in probing the transition from partonic to hadronic dynamics.

Figure 3 presents the first STAR measurements of mid-rapidity $(|y|<0.5) \phi$ meson spectra with a broad $p_{T}$ coverage for various collision centrality bins at selected BES energies $\sqrt{s_{N N}}=$ 7.7, 11.5, 19.6, 27, $39 \mathrm{GeV}$ [28]. Similar spectra were also obtained for $\Omega$ hyperon production in $\mathrm{Au}+\mathrm{Au}$ collisions. These results are used to examine features of formation mechanism in particle 


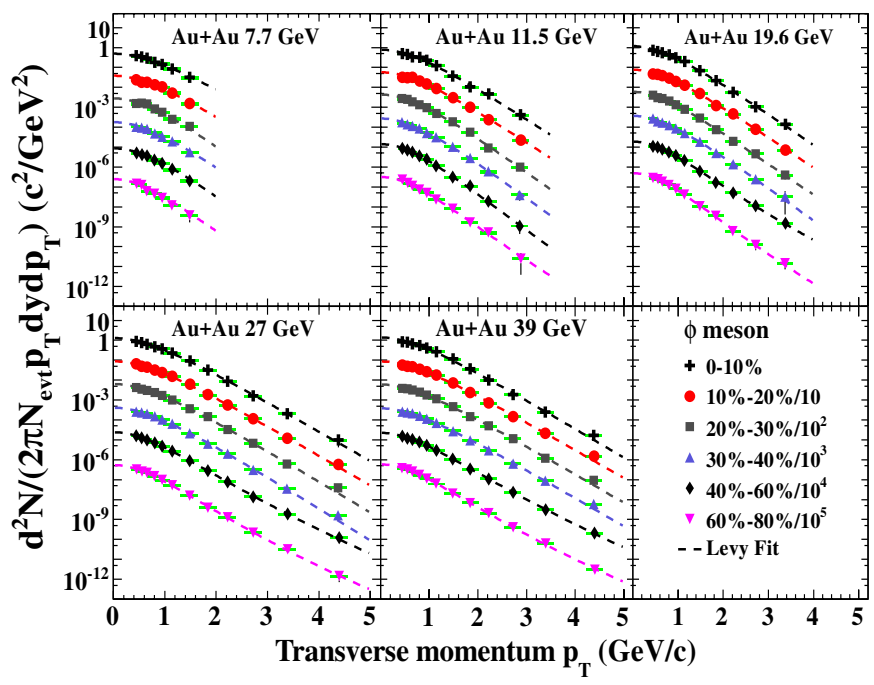

Figure 3. (Mid-rapidity $(|y|<0.5)$ transverse momentum spectra of $\phi$ mesons produced in Au+Au collisions at different centralities and energies $\left(\sqrt{s_{N N}}=7.7,11.5,19.6,27,39 \mathrm{GeV}\right)$ [28]. The green bands represent systematic errors. The dashed curves represent fits to the experimental data with a Levy function [29].

production and explore possible changes from bulk partonic coalescence to hadron dominated dynamics as the colliding energy decreases. Spectra were corrected for reconstruction efficiency and geometrical acceptance. The systematic errors $(10-16) \%$ at $\sqrt{s_{N N}}=11.5-39 \mathrm{GeV}$ and $(17-21) \%$ at $\sqrt{s_{N N}}=7.7 \mathrm{GeV}$ are mainly due to the different signal extraction techniques, and the reconstruction efficiency corrections were also taken into account.

The production of light nuclei with small binding energy such as (anti)deuterons, can be used to study the freeze-out properties and local baryon density in high-energy nuclear collisions. The azimuthal anisotropic results of protons and deuterons have shown that the coalescence is the dominant process for the light nuclei production at later stage of the evolution. The coalescence parameter $B_{A}$ for the production of nucleus $A$ is expressed via the proton and neutron yields as follows:

$$
\frac{d^{3} N_{A}}{d p_{A}^{3}}=B_{A}\left(\frac{d^{3} N_{p}}{d p_{p}^{3}}\right)^{Z}\left(\frac{d^{3} N_{n}}{d p_{n}^{3}}\right)^{(A-Z)}
$$

Here $Z$, and $A-Z$ are the number of protons and neutrons, respectively. The parameter characterizes the measure of the phase space density for nucleons. It is defined in a similar way for produced antinuclei. A difference between $B_{2}(A)$ and $B_{2}(\bar{A})$ may imply that baryon and anti-baryon freeze-out at different densities.

Results of systematic study of colliding energy, centrality, transverse momentum dependence of mid-rapidity deuteron and anti-deuteron production measured by the STAR experiment from $\mathrm{Au}+\mathrm{Au}$ collisions at $\sqrt{s_{N N}}=7.7,11.5,14.5,19.6,27,39$, and $200 \mathrm{GeV}$ [31] are presented in figure 4. Deuterons, protons and their anti-particles were identified using the time projection chamber (TPC) and time-of-flight detector (TOF). Proton and anti-proton yields are corrected for weak decays. It was found that the baryon density at mid-rapidity decreases with increasing energy, yield of $\bar{d}$ in- 

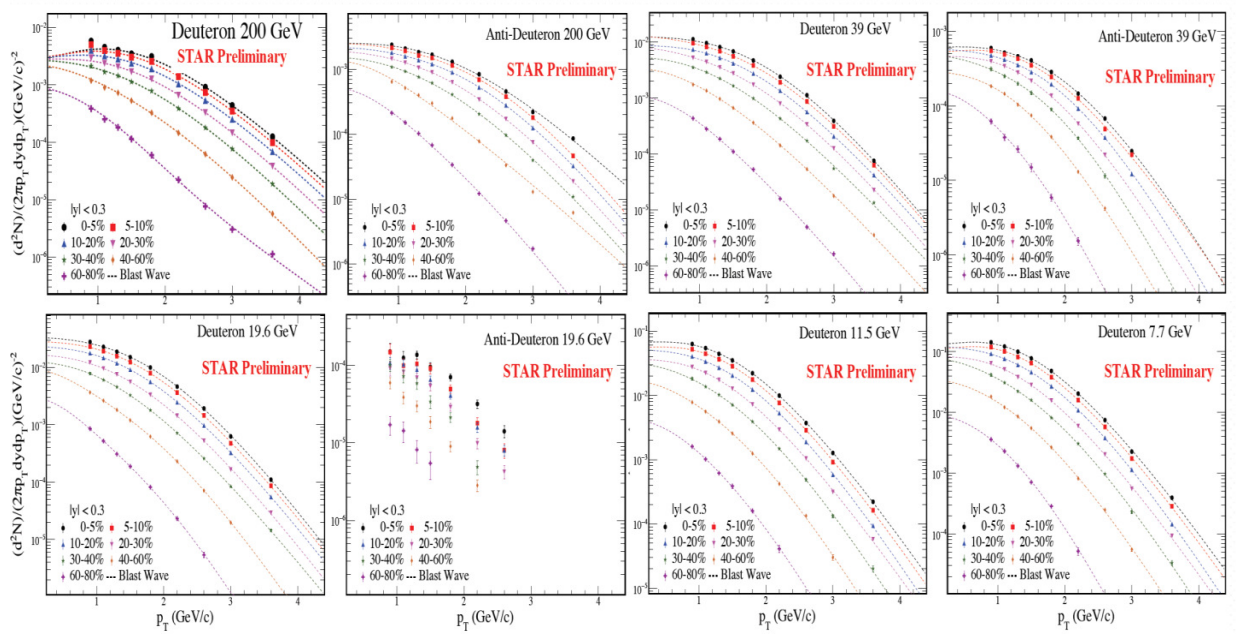

Figure 4. The transverse momentum distribution of $d$ and $\bar{d}$ production in Au+Au collisions at different centralities and energies $\sqrt{s_{N N}}=7.7,11.5,19.6,39 \mathrm{GeV}$ and $200 \mathrm{GeV}[31]$.

creases with increasing energy, the mean $p_{T}$ increases from peripheral to central collision and shows a weak energy dependence. The mean $p_{T}$ difference between $d$ and $\bar{d}$ was found to be small. The ratios $\bar{d} / d$ and $\bar{p} / p$ increase with increasing energy. The coalescence parameter $B_{2}(d)$ decreases as a collision energy increases, which shows that as energy decreases, the freeze-out volume for nucleons becomes smaller. The relative change $\left(B_{2}(d)-B_{2}(\bar{d})\right) / B_{2}(d)$ of the coalescence parameter shows little energy dependence, the average value is $0.29 \pm 0.14$, which might represent the iso-spin effect is not negligible in heavy-ion collision or particle and anti-particle have different freeze-out volume.

\subsection{Nuclear flow}

The medium created at top RHIC energies as reported in [1-4] passes through a partonic phase. Elliptic flow $v_{2}$ is one of the signal of collective phenomena of particle production

$$
\frac{d^{3} N_{A}}{d p_{A}^{3}}=\frac{1}{2 \pi p_{T}} \frac{d N_{A}}{d p_{T}}\left(1+\sum_{1}^{\infty} 2 v_{n} \cos \left(n\left(\phi-\Psi_{r}\right)\right)\right) .
$$

Therefore it is of interest to study how the signal $\left(v_{2}\right)$ evolves as a function of collision energy and centrality and type of produced particle when transverse particle densities change from low to high. Search for the switch from one to the other mechanism is one of the dramatic signatures of phase transition. If one scales the elliptic flow $v_{2} / n_{q}$ and transverse kinetic energy $E_{T} / n_{q}$ by the number $n_{q}$ of constituent quarks in the hadron, all particles fall on a common curve. Such scaling are explained by quark coalescence, or recombination models, which provide an intriguing framework for hadronization of bulk partonic matter at RHIC. The essential degrees of freedom at the hadronization seem to be effective constituent quarks which have developed a collective elliptic flow during the partonic evolution. Elliptic flow $v_{2}$ values for identified particles and anti-particles at midrapidity in $\mathrm{Au}+\mathrm{Au}$ collisions were measured by the STAR experiment in the Beam Energy Scan at RHIC at 


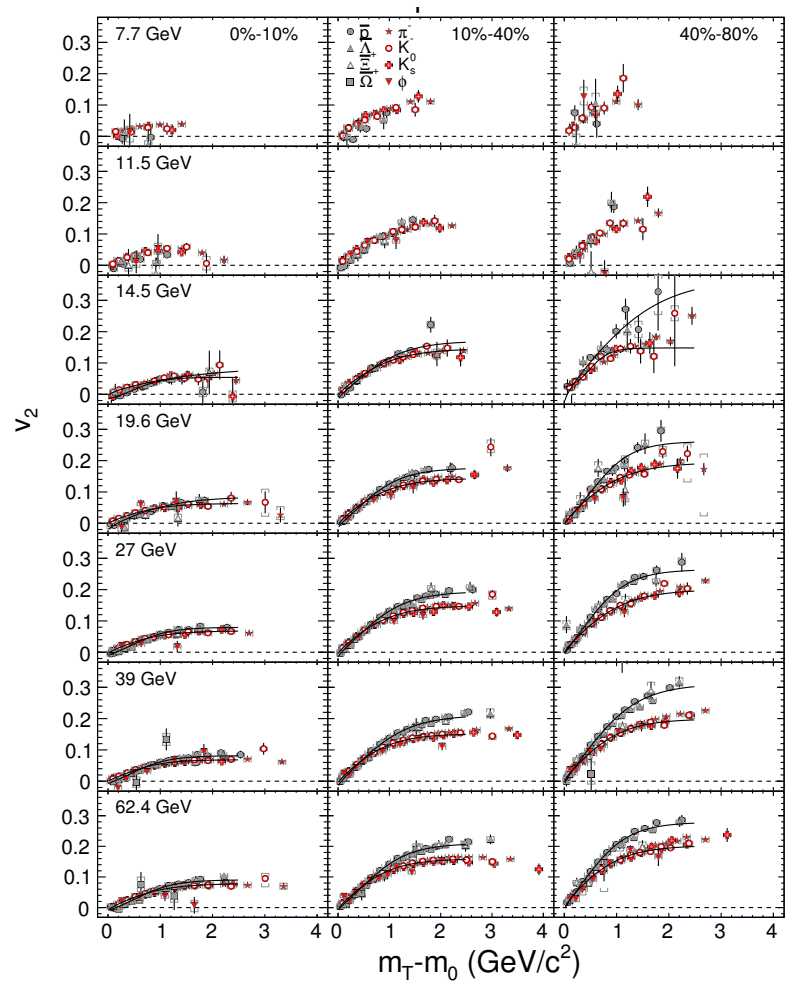

Figure 5. The elliptic flow $v_{2}$ of identified anti-particles $\pi^{-}, K^{-}, K^{0}, \bar{p}, \phi, \bar{\Lambda}, \bar{\Xi}^{+}, \bar{\Omega}^{+}$as a function of transverse kinetic energy $E_{T}=m_{T}-m_{0}$, for $(0-10) \%,(10-40) \%$, and $(40-80) \%$ central Au+Au collisions at $\sqrt{s_{N N}}=$ 7.7, 11.5, 14.5, 19.6, 27, 39, and $62.4 \mathrm{GeV}$ [30]. The lines show simultaneous fits to baryons and mesons. The systematic errors are shown by the hooked error bars.

$\sqrt{s_{N N}}=7.7,11.5,14.5,19.6,27,39 \mathrm{GeV}$ and $62.4 \mathrm{GeV}$ for three centrality classes [30]. A similar relative $v_{2}$ baryon-meson splitting for all centrality classes which is in agreement within $15 \%$ with the number-of-constituent quark scaling except at the lowest beam energies was observed. The larger $v_{2}$ for most particles relative to anti-particles shows a clear centrality dependence, with the largest difference for the most central collisions.

Figure 5 shows $v_{2}$ versus transverse kinetic energy $E_{T}=m_{T}-m_{0}$ of anti-particles $\pi^{-}, K^{-}, K^{0}, \bar{p}, \phi, \bar{\Lambda}, \bar{\Xi}^{+}, \bar{\Omega}^{+}$for three centrality ranges of $\mathrm{Au}+\mathrm{Au}$ collisions at $\sqrt{s_{N N}}=$ 7.7, 11.5, 14.5, 19.6, 27, 39, and $62.4 \mathrm{GeV}$ [30]. The splitting between baryons and mesons are found to be significant at $19.6 \mathrm{GeV}$ and higher energies, and marginally significant at $14.5 \mathrm{GeV}$. There is no observed splitting for all centralities at $11.5 \mathrm{GeV}$ and below. For these energies we are limited by the number of events and cannot draw a conclusion. For the $\phi$ meson at $14.5 \mathrm{GeV}$ there were not enough events to plot the centrality dependence. For every particle species, energy, and centrality, $v_{2}$ increases with increasing $E_{T}$. At $E_{T}>1 \mathrm{GeV}$ an onset of $v_{2}$ saturation can be observed. For the most central $(0-10) \%$ events the absolute baryon-meson splitting is significantly smaller compared to more peripheral collisions, partly because the values are smaller making the absolute difference smaller. 
Note, that if there were a perfect NCQ scaling, the ratio $v_{2}(B) / v_{2}(M)$ should be equal to 1.5 . It was found that the baryon-to-meson elliptic flow ratio for particles is higher than for anti-particles at all energies for $(0-10) \%$ and $(10-40) \%$ central collisions, but has no significant difference between particles and anti-particles for $(40-80) \%$. The ratio for anti-particles shows a centrality dependence which is increasing from central to peripheral collisions from about 1.3 to 1.6. But the ratio for particles does not show a significant centrality dependence. There is no significant beam energy dependence for the ratio of both particles and anti-particles for the points plotted, except for anti-particles at $(10-40) \%$ centrality. In addition, we can see from the ratio that NCQ scaling holds for particles at centralities of $(0-10) \%$ and $(10-40) \%$, but the ratio is slightly larger at $(40-80) \%$.

During the BES program, the STAR experiment has obtained results on $v_{2}$ at mid-rapidity $(|y|<$ 1.0) for light nuclei $d, t,{ }^{3} \mathrm{He}$ at $\sqrt{s_{N N}}=7.7,11.5,19.6,27,39,62.4$, and $200 \mathrm{GeV}$ and anti-nuclei $\bar{d}$ at $\sqrt{s_{N N}}=19.6,27,39,62.4$, and $200 \mathrm{GeV}$ and ${ }^{3} \bar{H} e$ at $\sqrt{s_{N N}}=200 \mathrm{GeV}$ [32]. It was found that the light (anti-)nuclei $v_{2}\left(p_{T}\right)$ shows a monotonic rise with increasing $p_{T}$, mass ordering at low $p_{T}$ and a reduction for more central collisions. It is observed that $v_{2}$ of nuclei and anti-nuclei are of similar magnitude at $\sqrt{s_{N N}}=39 \mathrm{GeV}$ and above. The difference $\Delta v_{2}$ between $d$ and $\bar{d}$ was found to follow the difference between $p$ and $\bar{p}$ as a function of collision energy. The flow $v_{2}$ for ${ }^{3} \mathrm{He}$ and $t$ nuclei shows similarity for all collision energies and in fact all the light-nuclei $v_{2}$ generally follow an atomic mass number scaling $\left(v_{2} / A\right)$. The scaling indicates that the coalescence of nucleons might be the underlying mechanism of light-nuclei formation in high energy heavy-ion collisions. It is noted in [32] that light nuclei could have also formed via coalescence of quarks when $v_{2}$ and $E_{T}$ are scaled by number of constituent quarks $n_{q}$ instead of mass number $A$. Although this process seems physically acceptable, the survival of light nuclei, with their low binding energies (a few $\mathrm{MeV}$ ), is highly unlikely under the high temperatures requisite for dissociating nucleons into quarks and gluons.

\subsection{Thermodynamic parameters}

The main aim of the RHIC BES program is to explore the phase diagram of nuclear matter created in heavy ion collisions [5]. It is assumed that the fireball produced in these collisions thermalizes rapidly leading to expansion and cooling of the nuclear system. Then the hadronization takes place and the particles get detected in the detectors.

Experimentally measured spectra of different particles over a wide range of centrality and collision energy can be used to determine thermodynamic parameters in the framework of the statistical thermal model [33-35] and the blast-wave model [36, 37]. The first model assumes that the produced system reached the thermodynamical equilibrium and statistical approach can be used to determine the chemical freeze-out temperature $T_{c h}$ and baryon chemical potential $\mu_{B}$. The second model considers that the local thermalization of particles at a kinetic freeze-out temperature $T_{k i n}$ and moving with a common radial flow velocity $\langle\beta\rangle$. The kinetic freeze-out temperature and average collective velocity parameters are extracted from blast-wave fits to the identified hadron spectra. STAR spectra of particles $\pi, K, p, \Lambda, \Xi$ and their anti-particles were used to determine the different particle ratios and to obtain the phase diagram in $\left\{T_{c h}, \mu_{B}\right\}$ and $\left\{T_{k i n},<\beta>\right\}$ planes [24-27].

Figure 6(a) demonstrates the dependence of $T_{c h}$ and $\mu_{B}$ on centrality (number of participants $N_{\text {part }}$ ) at different energies $\sqrt{s_{N N}}=7.7,11.5,14.5,19.6,27$, and $39 \mathrm{GeV}$. One can see from figure 6 (a) that $T_{c h}$ increases with the collision energy. The inverse behavior is observed for the chemical potential $\mu_{B}$. The centrality dependence of $\mu_{B}$ is seen as well. Figure 6(b) shows the dependence of spectra of $\pi^{+}, K^{+}, p$ particles and their anti-particles produced in $\mathrm{Au}+\mathrm{Au}$ collisions on the transverse momentum at different collision energies and centralities. These data have been used to extract the kinetic freezeout temperature $T_{k i n}$ and radial velocity $\langle\beta\rangle$. One can see that $\langle\beta\rangle$ decreases and $T_{k i n}$ increases from the central to peripheral collisions. An anti-correlation was observed between $T_{k i n}$ and $<\beta>$. 
The largest value of the collective velocity and lowest temperature is reached in the most central collisions at the highest collision energy.
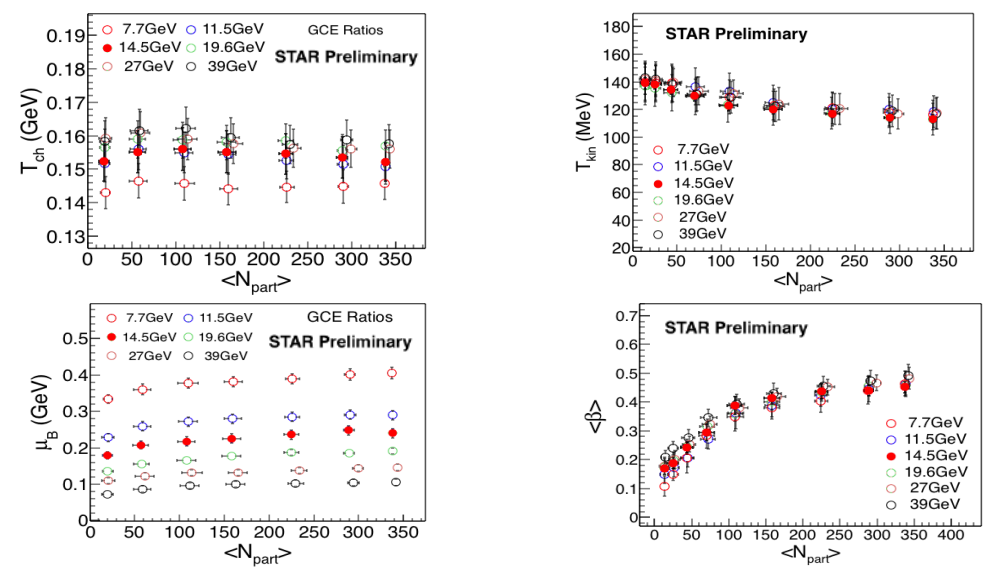

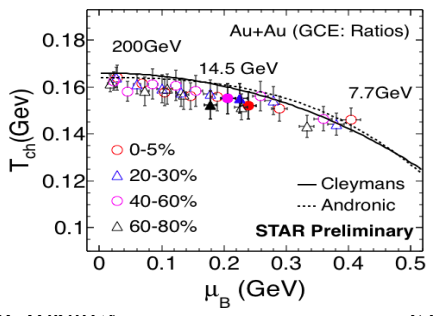

a)

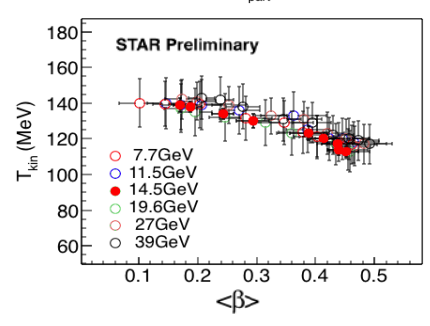

b)

Figure 6. Chemical $\left\{T_{c h}, \mu_{B}\right\}$ (a) and kinetic $\left\{T_{k i n},<\beta>\right\}$ (b) freeze-out thermodynamic parameters using the Grand-Canonical Ensemble of charged hadron production in $\mathrm{Au}+\mathrm{Au}$ collisions for different collision energy and centrality $[24,38-40]$.

\subsection{Self-similarity of hadron production in Au+Au collisions}

The idea of the Beam Energy Scan programs at RHIC is to span the phase diagram from the top RHIC energy to the lowest possible energy. It is known that the study of critical phenomena is most preferable in terms of dimensionless variables. Therefore self-similarity of hadron interactions could be assumed to be a very fruitful concept to study collective phenomena in the hadron and nuclear matter. Important manifestation of this concept is a notion of scaling itself. Scaling in general means self-similarity at different scales. The approach based on $z$-scaling suggested in $[41,42]$ was treated as manifestation of the self-similarity property of the structure of colliding objects (hadrons, nuclei), the interaction mechanism of their constituents, and the process of constituent fragmentation into real hadrons. We use the approach for analysis of the spectra of charged hadrons obtained by STAR in BES program [43]. The approach [41, 42] relies on a hypothesis about self-similarity of hadron interactions at a constituent level. The assumption of self-similarity transforms to the requirement of simultaneous description of transverse momentum spectra corresponding to different collision energies, rapidities, and centralities by the same scaling function $\psi(z)$ depending on a similarity parameter $z$. The scaling function for the collision of nuclei is expressed in terms of the experimentally measured inclusive 
invariant cross section $\left(E d^{3} \sigma / d p^{3}\right)$, the multiplicity density $(d N / d \eta)$, and the total inelastic cross section $\left(\sigma_{\text {in }}\right)$, as follows:

$$
\psi(z)=-\frac{\pi}{(d N / d \eta) \sigma_{\text {in }}} J^{-1} E \frac{d^{3} \sigma}{d p^{3}} .
$$

Here $J$ is the Jacobian for the transformation from $\left\{p_{T}^{2}, y\right\}$ to $\{z, \eta\}$. The function $\psi$ is interpreted as a probability density to produce an inclusive particle with the corresponding value of $z$. The similarity parameter $z$ is expressed via momentum fractions $\left(x_{1}, x_{2}, y_{a}, y_{b}\right)$, multiplicity density, and parameters $\delta_{1,2}, \epsilon_{a, b}, c$, as follows:

$$
z=z_{0} \Omega^{-1}
$$

where $z_{0}=\sqrt{s_{\perp}} /\left[\left(d N_{c h} /\left.d \eta\right|_{0}\right)^{c} m_{N}\right], \Omega=\left(1-x_{1}\right)^{\delta_{1}}\left(1-x_{2}\right)^{\delta_{2}}\left(1-y_{a}\right)^{\epsilon_{a}}\left(1-y_{b}\right)^{\epsilon_{b}}$. Here $m_{N}$ is a nucleon mass. The quantity $\sqrt{s_{\perp}}$ is the transverse kinetic energy of the binary subprocess consumed on the production of the inclusive particle with mass $m_{1}$ and its counterpart with mass $m_{2}$. The constituents of the incoming nuclei carry fractions $x_{1}, x_{2}$ of their momenta. The inclusive particle carries the momentum fraction $y_{a}$ of the scattered constituent. The parameters $\delta_{1,2}$ and $\epsilon_{a, b}$ describe structure of the colliding nuclei and fragmentation process, respectively. The parameter $c$ is interpreted as a "specific heat" of the created medium. Simultaneous description of different spectra with the same $\psi(z)$ puts strong constraints on the values of these parameters, and thus allows for their determination. It was found that $\delta_{1,2}$ and $c$ are constant at high energies, $\epsilon_{a, b}$ depends on multiplicity and $m_{1}=m_{2} \equiv m$. For the obtained values of $\delta_{1,2}, \epsilon_{a, b}$ and $c$, the momentum fractions are determined to minimize the resolution $\Omega^{-1}\left(x_{1}, x_{2}, y_{a}, y_{b}\right)$ with the constraint $\left(x_{1} P_{1}+x_{2} P_{2}-p / y_{a}\right)^{2}=\left(x_{1} M_{1}+x_{2} M_{2}+m / y_{b}\right)^{2}$, which has sense of the momentum conservation law of a constituent subprocess. The fractal dimension of the nucleus is expressed in terms of the nucleon fractal dimension $(\delta)$ and the atomic number of nucleus $(A)$ as follows, $\delta_{A}=A \delta$. The fragmentation fractal dimension in nucleus+nucleus collisions is parameterized in the form: $\epsilon_{A A}=\epsilon_{0}(d N / d \eta)+\epsilon_{p p}$. It depends on multiplicity density and nucleon fragmentation dimension $\left(\epsilon_{p p}=0.2\right)$. The negatively charged hadron spectra measured by STAR in Au+Au collisions at $\sqrt{s_{N N}}=7.7,11.5,19.6,27,62.4$ and $200 \mathrm{GeV}$ at mid-rapidity $|\eta|<0.5$ as a function of transverse momentum $p_{T}$ for different centrality classes of $(0-5) \%,(5-10) \%$,

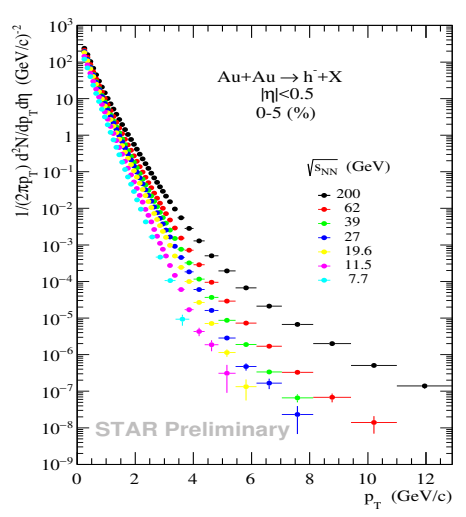

a)

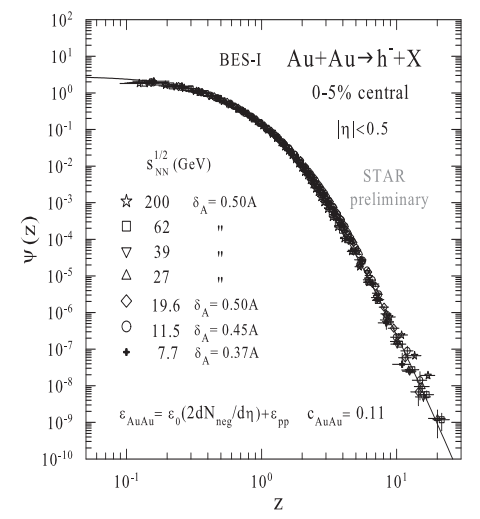

b)

Figure 7. The dependence of the spectra of negatively charged particles produced in the most central $\mathrm{Au}+\mathrm{Au}$ collisions at energy $\sqrt{s_{N N}}=7.7,11.5,19.6,27,39,62.4$, and $200 \mathrm{GeV}$ in the range $|\eta|<0.5$ [43] on the transverse momentum (a) and the corresponding dependence of scaling function $\psi(z)$ on the self-similarity parameter $z$ (b). 
$(10-20) \%,(20-40) \%,(40-60) \%,(60-80) \%$ were presented in [43]. The transverse momentum spectra for these energies and for most central events $(0 \%-5 \%)$ are shown in figure $7(\mathrm{a})$. The distributions are measured over a wide momentum range $0.2<p_{T}<12 \mathrm{GeV} / \mathrm{c}$. As we can see the data presentation in terms of dimensional quantities demonstrates a strong dependence on the scale. Figure 7 (b) shows scaling function $\psi(z)$ as a function of self-similarity parameter $z$ for the data presented in figure 7(a). We observe a "collapse" of the data onto a single curve. The dependence demonstrates the universality of the shape of the scaling curve, the constancy of the fractal dimensions of the nuclei and the fragmentation process in the range $\sqrt{s_{N N}}>19.6 \mathrm{GeV}$. The found regularity can be treated as manifestation of the self-similarity of the structure of colliding objects, interaction mechanism of their constituents, and processes of fragmentation of these constituents into real registered particles. It is assumed that the discontinuity of the model parameters ("heat capacity" and fractal dimensions) indicates on the existence of critical phenomena in a nuclear matter.

\section{Heavy flavor}

Charm and beauty quarks are created predominantly via initial hard scatterings in nucleon-nucleon collisions and the production rate is calculable with perturbative QCD techniques. The large masses are expected to be retained during the interactions with the nuclear medium. Heavy quarks are therefore predicted to be sensitive to transport and other properties of the early stages of the system when the QGP is expected to exist.

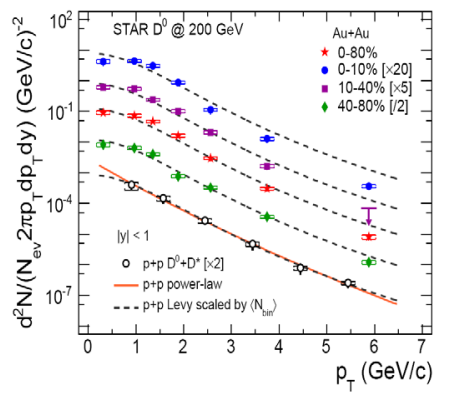

a)

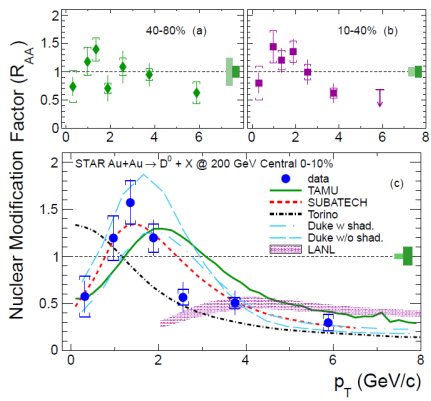

b)

Figure 8. The dependence of the differential invariant yield (a) and the nuclear modification factor $R_{A A}$ (b) of $D^{0}$ meson production in $\mathrm{Au}+\mathrm{Au}$ collisions at $\sqrt{s_{N N}}=200 \mathrm{GeV}$ on the transverse momentum $p_{T}$ for different centralities. The curves (left panel) are number-of-binary collisions scaled Levy functions from fitting to the $\mathrm{p}+\mathrm{p}$ result (open circles) [51]. The arrow denotes the upper limit with $90 \%$ confidence level of the last data point for $(10-40) \%$ collisions. The systematic uncertainties are shown as square brackets. [50].

Energetic heavy quarks were predicted to lose less energy via gluon radiation than light quarks when they traverse the QGP. Initial RHIC and LHC measurements, however, show similar suppression at high transverse momentum, $p_{T}$, in central $\mathrm{A}+\mathrm{A}$ collisions (see [50] and references therein). This has led to the reconsideration of the effects of heavy-quark collisional energy loss and requires follow-up measurements. Heavy-quark collective motion can provide experimental evidence for bulk medium thermalization. Model calculations show that interactions between heavy quarks and the QGP are sensitive to the drag/diffusion coefficients of the medium. These can be related to the shear viscosityto-entropy ratio and other transport properties. Therefore, measurements of heavy-quark production at low and intermediate $p_{T}$ are of particular relevance to these issues, and also for the interpretation of the 
charmonia production in heavy-ion collisions. In elementary collisions, heavy quarks are expected to hadronize mainly through hard fragmentation. In high energy heavy-ion collisions, the large charmpair abundance could increase the coalescence probability. The coalescence of charm with a light quark from the medium with a large radial flow may introduce a $p_{T}$-dependent modification to the observed charmed hadron spectrum compared to that from fragmentation. Furthermore, this may lead to a baryon-to-meson enhancement for charmed hadrons similar to that observed for light flavor hadrons.

Figure 8(a) shows the first measurement of the spectra (solid symbols) of $D^{0}$ meson production over a transverse momentum range $0.0<p_{T}<6.0 \mathrm{GeV} / \mathrm{c}$ in $\mathrm{Au}+\mathrm{Au}$ collisions at $\sqrt{s_{N N}}=200 \mathrm{GeV}$ for different centrality bins [50]. The measurement was performed via invariant-mass reconstruction of the hadronic decay channel $D^{0} \rightarrow K^{-}+\pi^{+}$and its charge conjugate. The $p p$ data, shown as open circles, contain $D^{0}$ data for $p_{T}<2.0 \mathrm{GeV} / \mathrm{c}$ and $D^{*}$ data for $p_{T}>2.0 \mathrm{GeV} / \mathrm{c}$. The main subsystems used for the $D^{0}$ analysis are the TPC and TOF. All measurements are presented as an average of $D^{0}$ and $\bar{D}^{0}$ yields at midrapidity $(|y|<1)$. The nuclear modification factor $R_{A A}$ for $D^{0}$ meson production calculated as the ratio between the $p_{T}$ spectrum in $\mathrm{Au}+\mathrm{Au}$ collisions for the centrality bins of $(40-80) \%,(10-40) \%$, and $(0-10) \%$ to the Levy function fit to the $p+p$ data is shown in figure 8(b). The statistical and systematic uncertainties are shown by the vertical lines and brackets, respectively. Strong suppression is observed in the most-central collisions for $p_{T}>2.5 \mathrm{GeV} / \mathrm{c}$, while no evidence is found for suppression in peripheral collisions. In $(0-10) \%$ collisions, the suppression level is around 0.5 for $p_{T}>3 \mathrm{GeV} / \mathrm{c}$, which is consistent with both the measurements of electrons from heavy-flavor hadron decays and the light hadrons. These results as noted in [50] indicate that charm quarks lose significant energy in the hot and dense medium produced in the central Au+Au collisions at RHIC and support the coalescence hadronization of $D$ meson production.

The STAR experiment has completed data taking with two new detectors for heavy flavor studies, the Heavy Flavor Tracker (HFT) [44] and Muon Telescope Detector (MTD) [45-49].

Figure 9 demonstrates the transverse momentum spectra (blue symbols)(a) and nuclear modification factor (black symbols) (b) for $D^{0}$ meson production in $\mathrm{Au}+\mathrm{Au}$ collisions obtained by using the TPC, TOF and HFT [52]. The spectra are measured up to $6.5 \mathrm{GeV} / \mathrm{c}$ and are in a good agreement with

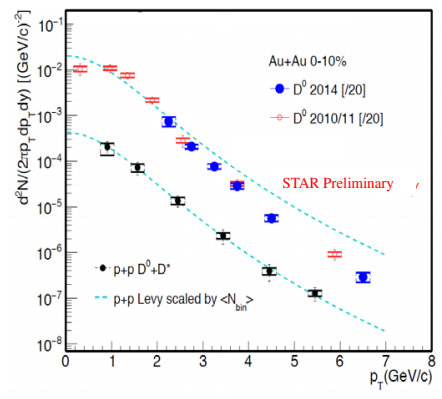

a)

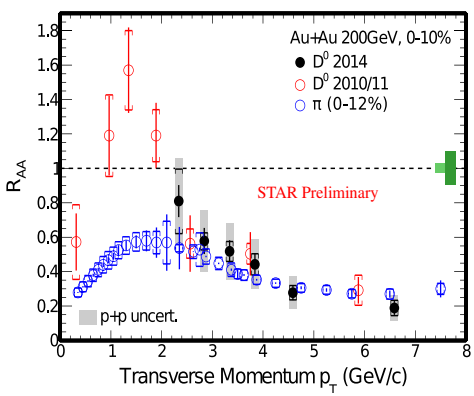

b)

Figure 9. The dependence of the differential invariant yield (a) and the nuclear modification factor $R_{A A}$ (b) of $D^{0}$ meson production in $\mathrm{Au}+\mathrm{Au}$ collisions at $\sqrt{s_{N N}}=200 \mathrm{GeV}$ on the transverse momentum $p_{T}$ for $(0-10) \%$ central collisions [52]. The curves (left panel) are number-of-binary collision scaled Levy functions from fitting to the $\mathrm{p}+\mathrm{p}$ data (black symbols) [51]. Data taken with and without the HFT are shown by blue and red symbols on the left panel (a) and black and red symbols on the right panel (b). The systematic uncertainties are shown as square brackets. 


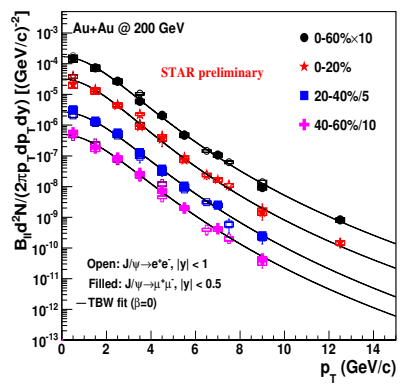

a)

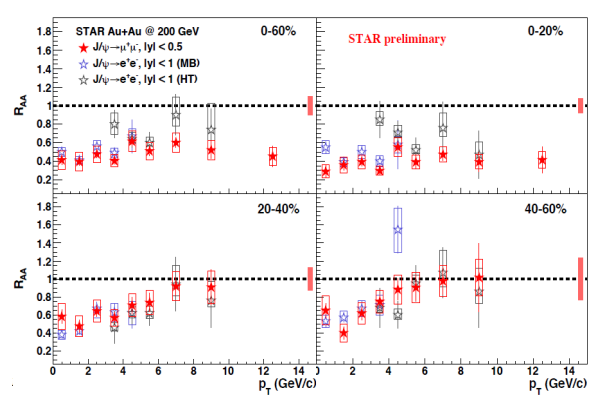

b)

Figure 10. The invariant yield of $J / \psi$ scaled by the branching ratio $B_{l l}$ (a) and the nuclear modification factor $R_{A A}$ (b) as a function of $p_{T}$ for different centralities in the di-muon (filled symbols ) [53] and in the di-electron (open symbols) [56] channels.

the previous data [50] shown by red symbols. The value of $R_{A A}$ is larger than 1 for $p_{T} \sim 1.5 \mathrm{GeV} / \mathrm{c}$. It indicates on charm coalescence with a radially flowing bulk medium. Significant suppression of $D^{0}$ production in most central $(0-10) \% \mathrm{Au}+\mathrm{Au}$ collisions for $p_{T}>2.5 \mathrm{GeV} / \mathrm{c}$ is observed. The value of $R_{A A}$ at high $p_{T}$ is found to be the same $(\sim 0.2)$ as for $\pi$ mesons shown by blue symbols in figure $9(\mathrm{~b})$. This result indicates on strong charm-medium interaction.

Using the MTD detector, STAR has studied $J / \psi$ production in Au+Au collisions at $\sqrt{s_{N N}}=$ $200 \mathrm{GeV}$ [53]. Figure 10(a) shows the invariant yield of $J / \psi$ in $\mathrm{Au}+\mathrm{Au}$ collisions at $\sqrt{s_{N N}}=200 \mathrm{GeV}$ for different collision centralities. As seen from figure 10(a) the new results in the di-muon decay channel measured in the range $0<p_{T}<15 \mathrm{GeV} / \mathrm{c}$ are consistent with previous results in the dielectron decay channel [54-56]. The Tsallis Blast-Wave fits to di-electron results shown by solid lines assume zero velocity for $J / \psi$.

Figure 10(b) shows the nuclear modification factor $R_{A A}$ for di-muon (red star) and di-electron (open black star) channels as a function of transverse momentum for different centralities

$$
R_{A A}=\frac{\left(\sigma_{\text {inel }}\right)}{\left(N_{\text {coll }}\right)} \frac{\left(d^{2} N_{A A} / d p_{T} d y\right)}{\left(d^{2} \sigma_{p p} / d p_{T} d y\right)} .
$$

We see strong suppression of $R_{A A}$ at low $p_{T}<3-4 \mathrm{GeV} / \mathrm{c}$. The mechanism of dissociation plays a significant role in this $p_{T}$ range. For middle centralities $(20-40) \%$ and $(40-60) \%$ the ratio $R_{A A}$ increases with $p_{T}$ and reaches unity at $p_{T}=8-9 \mathrm{GeV} / \mathrm{c}$. For most central Au+Au collisions $(0-20) \%$ suppression is large up to $p_{T} \simeq 12 \mathrm{GeV} / \mathrm{c}$. The dependence of $R_{A A}$ on transverse momentum is consistent with di-electron channel results for the entire $p_{T}$ range within uncertainties in all centralities.

\section{Fixed target mode at STAR}

As it was noted above the RHIC BES-I program [5] was proposed to search for the possible critical point and to study the nature of the phase transition between hadronic and partonic phases of matter. The first goal of the BES-I program, namely the evidence for the disappearance of signatures of quark-gluon phase observed at the RHIC top energy, is already qualitatively reached with the results of BES-I. The original design of RHIC optimized the luminosity at $\sqrt{s_{N N}}=200 \mathrm{GeV}$. At lower energies, the beam is not as well-focused and the collision rate is low. The STAR BES Phase II White 


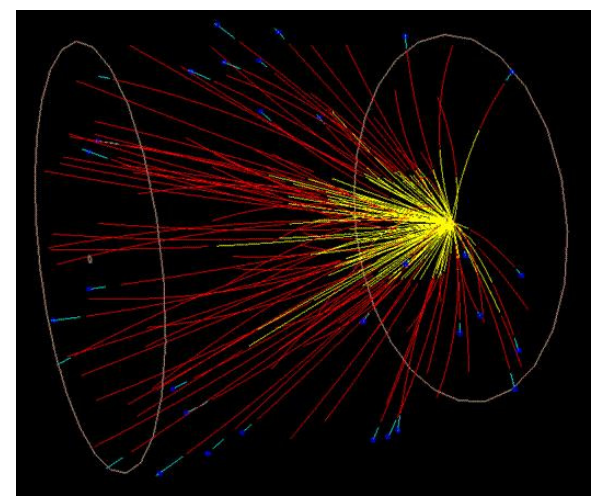

Figure 11. Central $\mathrm{Au}+\mathrm{Au}$ collision event at $\sqrt{s_{N N}}=3.9 \mathrm{GeV}$ in the fixed target mode. The reconstructed tracks from an interaction between a nucleus in the beam halo and a gold nucleus [59].

Paper details the motivation and the plans to return to energy scans at low RHIC energy in years 2018 and 2019 [16]. In order to provide adequate intensity for BES phase II, the RHIC Collider Accelerator Division (CAD) has been undertaking an upgrade with electron cooling [70-72]. It is expected that with electron cooling, the luminosity will increase by a factor of $2-5$ at $\sqrt{s_{N N}}=7.7 \mathrm{GeV}$ in the collider mode. Using interactions between beam nuclei and a stationary gold target [58-61] the feasibility has been demonstrated to study $\mathrm{Au}+\mathrm{Au}$ events at center-of-mass energies below $7.7 \mathrm{GeV}$ (up to $3.0 \mathrm{GeV}$ ) during the BES phase II. The results [57] obtained by NA49 Collaboration at CERN support the onset of deconfinement at a collision energy of $7.7 \mathrm{GeV}$. Therefore the enlarged statistics of BES phase II would allow us to improve on accuracy of earlier results, and to precisely determine at which collision energy QGP signatures disappear. The QGP signatures disappear at the low end of the BES-I energy range. An internal fixed-target mode will be used to access collision energies below $7.7 \mathrm{GeV}$.

Figure 11 shows the reconstructed tracks in $\mathrm{Au}+\mathrm{Au}$ collision at $\sqrt{s_{N N}}=3.9 \mathrm{GeV}$ from an event associated with the gold target. Figure 12 demonstrates the spectra (a) and ratio (b) of $\pi^{+}$and $\pi^{-}$ meson production in $\mathrm{Au}+\mathrm{Al}$ collisions at energy $\sqrt{s_{N N}}=3.0,3.5,4.0 \mathrm{GeV}$.

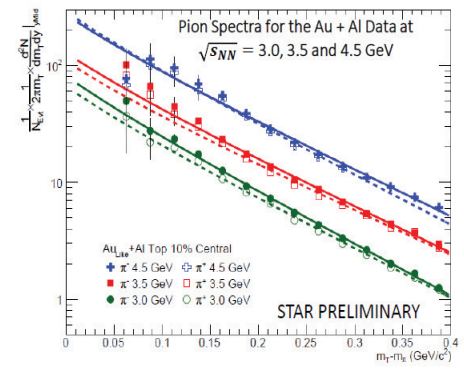

a)

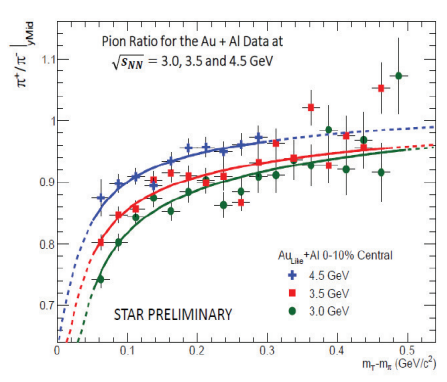

b)

Figure 12. Spectra (a) and ratio (b) of $\pi^{+}$and $\pi^{-}$meson production in Au+Al collisions in fixed target mode at energy $\sqrt{s_{N N}}=3.0,3.5,4.0 \mathrm{GeV}$ [58-61]. 


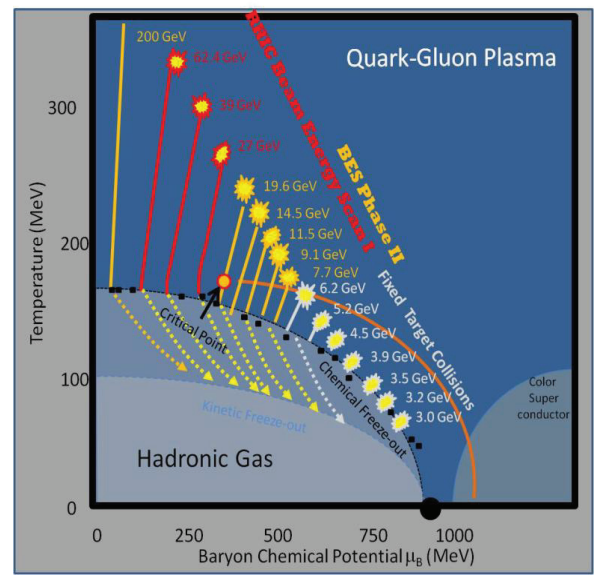

Figure 13. The supposed phase diagram of nuclear matter which is created in central $\mathrm{Au}+\mathrm{Au}$ collisions at over a range $\sqrt{s_{N N}}=3-200 \mathrm{GeV}$ in the $\left(T_{c h}, \mu_{B}\right)$ plane.

It is expected that the fixed-target program will enable STAR to make key measurements related to the phase diagram of nuclear matter below the reported onset of deconfinement at $\sqrt{s_{N N}}=7.7 \mathrm{GeV}$.

Figure 13 shows the reach of RHIC's BES program in the $\left(T_{c h}, \mu_{B}\right)$ plane at energies $\sqrt{s_{N N}}=$ $3.0-200 \mathrm{GeV}$. This choice of energies provides almost uniform coverage of the unknown $\left\{T_{c h}, \mu_{B}\right\}$ region and may allow us to narrow down an area of interest for further study. The range of baryon chemical potential $\left(\mu_{B}\right)$ changes for BES-II energy in the collider and fixed target modes is shown in table 1 . One can see that values of $T_{c h}$ and $\mu_{B}$ cover a range from $420 \mathrm{MeV}$ to $205 \mathrm{GeV}$ at $\sqrt{s_{N N}}=7.7$ and $20 \mathrm{GeV}$ in the collider mode and from $750 \mathrm{MeV}$ to $420 \mathrm{MeV}$ at $\sqrt{s_{N N}}=3.0$ and $7.7 \mathrm{GeV}$ in the fixed target mode, respectively. Note, that this range is a larger range than at any other heavy-ion facility.

Table 1. The $\mu_{B}$ values are estimated from the systematics of central collisions at BES-II energies [16].

\begin{tabular}{|c|c|c|c|c|c|}
\hline $\begin{array}{l}\text { Collider } \\
\text { energy } \\
(\mathrm{GeV})\end{array}$ & $\begin{array}{l}\text { Fixed } \\
\text { Target } \\
\text { energy } \\
(\mathrm{GeV})\end{array}$ & $\begin{array}{l}\text { Center- } \\
\text { of-mass } \\
\text { rapidity }\end{array}$ & $\begin{array}{l}\text { Single } \\
\text { beam } \\
\text { energy } \\
(\mathrm{GeV})\end{array}$ & $\begin{array}{l}\text { Chemical } \\
\text { potential } \\
\mu_{B}(\mathrm{MeV}) \\
(\text { collider mode })\end{array}$ & $\begin{array}{l}\text { Chemical } \\
\text { potential } \\
\mu_{B}(\mathrm{MeV}) \\
\text { (fixed target mode) }\end{array}$ \\
\hline 19.6 & 4.47 & 1.52 & 8.87 & 205 & 589 \\
\hline 17.2 & 4.21 & 1.46 & 7.67 & 230 & 608 \\
\hline 14.5 & 3.90 & 1.37 & 6.32 & 250 & 633 \\
\hline 13.0 & 3.72 & 1.32 & 5.57 & 288 & 649 \\
\hline 11.5 & 3.53 & 1.25 & 4.82 & 315 & 666 \\
\hline 9.1 & 3.19 & 1.13 & 3.62 & 370 & 699 \\
\hline 7.7 & 2.98 & 1.05 & 2.92 & 420 & 721 \\
\hline
\end{tabular}




\section{STAR upgrades for BES-II}

The limited event statistics from BES-I has already allowed significant progress to be made toward the goals that were established at the outset of this program [5]. There are clear indications that hadronic interactions dominate at the lower BES energies and several observables associated with the formation of a partonic phase at top RHIC energy show indications of turn-off. The successful performance of the BES-II program [16] and further understanding of the phase diagram of QCD matter is only possible with the STAR upgrade [62, 63], in combination with the higher RHIC luminosities due to electron cooling [70, 71]. New systems - the internal Time Projection Chamber (iTPC) and Event Plane Detector (EPD), will increase STAR's mid-rapidity acceptance, event plane resolution, and centrality determination. The BES phase II program requests $\mathrm{Au}+\mathrm{Au}$ collisions at $\sqrt{s_{N N}}=19.6,14.6,11.5$ and $7.7 \mathrm{GeV}$ after electron cooling upgrade. The fixed gold target will allow collisions at low energy $\sqrt{s_{N N}}=4.5,4.0,3.5$, and $3.0 \mathrm{GeV}$, respectively. These events can be triggered upon and recorded without reducing the number of collider events.

The inner TPC upgrade [64-66] will provide the increase of transverse momentum acceptance from $p_{T}>125 \mathrm{MeV}$ to $p_{T}>60 \mathrm{MeV} / \mathrm{c}$, improve $d E / d x$ resolution from $7.5 \%$ to $6.2 \%$, and increase acceptance from $|\eta|<1$ to $|\eta|<1.5$. The end cap Time-Of-Flight [67] will provide PID of $\pi / K / p$ for the whole iTPC acceptance in the collider and fixed target modes. The Event Plane Detector [68] will provide large $\eta$ coverage $2.1<|\eta|<5.1$ compared to TPC $(|\eta|<1.0)$, high $\eta$ (radial, 16) and azimuthal (24) segmentation, good timing resolution ( $1 \mathrm{~ns})$, and additional mid-rapidity independent event plane and centrality determination independent from TPC. It will be also used as a trigger detector for BES-II.

So the STAR upgrade (iTPC+eTOF+EPD) is crucial to extend energy reach to overlap/complementary AGS/FAIR/NICA/JPARC and provide the unprecedented coverage and PID for critical point search in BES-II.

\section{Conclusions}

The study of features of the phase diagram of the nuclear matter created in collisions of nuclei at RHIC is one of the major scientific tasks in heavy ion physics. These are location of a possible critical point and phase boundaries, determination of type of the phase transitions. The ability of RHIC to scan a wide range of beam energies $\left(\sqrt{s_{N N}}=7.7-200 \mathrm{GeV}\right)$, beam and probe species provides a unique opportunity for the exploration of the phase diagram. RHIC has completed Phase-I of the beam energy scan program with center-of-mass beam energies $\sqrt{s_{N N}}=7.7,11.5,14.5,19.6,27$ and $39 \mathrm{GeV}$. Some of resent results obtained by the STAR Collaboration were presented in the report. Among them is the dependence of the nuclear modification factor $R_{C P}$ on transverse momentum $p_{T}$ for different collision energy $\sqrt{s_{N N}}$. The results demonstrate the evolution of suppression of particle yields with $\sqrt{s_{N N}}$. It indicates on strong modification of a mechanism of the hadron production by the nuclear medium.

It was found that the spectra of different particle $(\pi, p, K, J / \psi, \phi, \Lambda, \Xi, \Omega, d, H e, \ldots)$ and antiparticle production at BES energy and different centrality of collisions over a wide range of $p_{T}$ show strong sensitivity to medium environment and flavor content of produced particles. Indication of the nucleon coalescence mechanism for production of light nuclei $\left(d,{ }^{3} \mathrm{He},{ }^{4} \mathrm{He}\right)$ and their anti-nuclei was supported.

The dependence of elliptic flow on the transverse momentum $p_{T}$ for particles $\pi, p, K, \phi, \Lambda, \Xi, \Omega$ and their anti-particles found at BES energies supports the hypothesis on parton collectivity and reveals features of the number constituent quark scaling in $\mathrm{Au}+\mathrm{Au}$ collisions firstly observed at $200 \mathrm{GeV}$. 
The spectra of strange and non-strange particles and anti-particles and their ratios measured by STAR over a wide range of centrality and collision energy were used to determine the thermodynamic parameters in the framework of the statistical thermal model and blast-wave model. The results allow us to scan the phase diagram of nuclear matter over a wide range in $T_{c h}, \mu_{B}$ and $T_{k i n},<\beta>$ planes and to verify a scenario of chemical and kinetic freeze-out.

Using new detectors - Heavy Flavor Tracker and Muon Telescope Detector, the STAR Collaboration has performed the measurements of $D$ and $J / \psi$ mesons production in Au+Au collisions at $\sqrt{s_{N N}}=200 \mathrm{GeV}$. The dependence of $R_{A A}$ on the transverse momentum was found to be consistent with di-electron channel results and confirm strong suppression of particle yields for most central $\mathrm{Au}+\mathrm{Au}$ collisions at large $p_{T}$ as it has been observed for light flavor particles.

The STAR BES phase II sets out the physics case for an in-depth study of energies below $20 \mathrm{GeV}$ with typically 20 times the statistics as in the same energy region in BES-I. It can be done in the collider and fixed target modes. Low-energy RHIC electron cooling is an essential point in the collider mode. The fixed target program will extend BES-II physics reach to the region below the onset of deconfinement down to energy $\sqrt{s_{N N}}=3 \mathrm{GeV}$.

In accordance to general theory of critical phenomena [73, 74] the "scaling" and "universality" are concepts developed to understand the behavior and properties of multiparticle systems. Scaling means that systems near critical point exhibiting self-similar properties are invariant under transformation of scale. According to universality, quite different systems behave in a remarkably similar fashion near the respective critical points. It is expected that experimental characteristics of heavy ion collisions such as fluctuation and correlation functions should reveal a power behavior, demonstrate a strong enhancement and be described by the critical exponents near critical point. Therefore search for new scaling laws of the particle production in $\mathrm{Au}+\mathrm{Au}$ collisions is of interest to verify the fundamental physical principles such as self-similarity [75]. We consider that the collider and fixed target modes with new detector systems (HFT, MTD, iTPC, EPD, eTOF) significantly improve the capabilities of STAR for BES-II to detect key features of QCD phase diagram.

\section{References}

[1] J. Adams et al. (STAR collabotration), Nucl. Phys. A 757, 102 (2005)

[2] K. Adcox et al. (PHENIX collabotration), Nucl. Phys. A 757, 184 (2005)

[3] B. B. Back et al.(PHOBOS collabotration), Nucl. Phys. A 757, 28 (2005)

[4] I. Arsene et al. (BRAHMS collabotration), Nucl. Phys. A 757, 1 (2005)

[5] M. M. Aggarwal et al. (STAR Collaboration), "An Experimental Exploration of the QCD Phase Diagram: The Search for the Critical Point and the Onset of Deconfinement", arXiv:1007.2613

[6] L. Kumar (for STAR Collaboration), Nucl. Phys. A 830, 275C (2009)

[7] L. Kumar (for STAR Collaboration), Nucl. Phys. A 862-863, 125 (2011)

[8] B. Mohanty (for STAR Collaboration), Nucl. Phys. A 830, 899C (2009)

[9] B.I. Abelev et al. (STAR Collaboration), Phys. Rev. C 81, 024911 (2010)

[10] B.I. Abelev et al. (STAR Collaboration), Phys. Lett. B 655, 104 (2007)

[11] J. Adams et al. (STAR Collaboration), Phys. Rev. Lett. 95, 122301 (2005)

[12] A. Adare et al. (STAR Collaboration), Phys. Rev. Lett. 98, 162301 (2007)

[13] B.I. Abelev et al., Phys. Rev. C 80, 064912 (2009)

[14] Y. Aoki et al., Nature 443 675, (2006). S. Ejiri, Phys. Rev. D 78, 074507 (2008). E.S. Bowman and J. I. Kapusta, Phys. Rev. C 79, 015202 (2009). M.A. Stephanov, Int. J. Mod. Phys. A 20, 4387 (2005). S. Gupta et al., Science 332, 1525 (2011) 
[15] S.Sharma, "Recent Lattice QCD Results for RHIC BES", RHIC \& AGS Annual Users' Meeting, Upton, BNL, January 7-10, 2016. https://www.bnl.gov/aum2016/

[16] STAR Collaboration, "Studying the Phase Diagram of QCD Matter at RHIC". https://drupal.star.bnl.gov/STAR/starnotes/public/sn0598

[17] K. Ackermann et al. (STAR Collaboration), Nucl. Instrum. Meth. A 499, 624 (2003)

[18] M. Anderson et al. (STAR Collaboration), Nucl. Instrum. Meth. A 499, 659 (2003)

[19] W. Llope et al. (STAR Collaboration), Nucl. Instrum. Meth. B 241, 306 (2005)

[20] F. Geurts et al., Nucl. Instrum. Meth. A 533, 60 (2004). Y. Wang et al., Nucl. Instrum. Meth. A 613, 200 (2010)

[21] M.A.C. Lamont (for the STAR Collaboration), J. Phys. Conf. Ser. 50, 192 (2006)

[22] S. Horvat (for the STAR Collaboration), arXiv:1303.7260, arXiv:1601.01644

[23] S. Horvat (for the STAR Collaboration), "Charged Hadron Nuclear Modification Factors in the Beam Energy Scan Data from STAR", 8th International Workshop on Critical Point and Onset of Deconfinement, PoS(CPOD 2013)002, March 11-15, 2013, Napa, California, USA

[24] V. Bairathi (for the STAR Collaboration), Nucl. Phys. A 956, 292 (2016)

[25] L. Kumar (for the STAR Collaboration), Nucl. Phys. A 862-863, 125 (2011)

[26] L. Kumar (for the STAR Collaboration), Nucl. Phys. A 904-905, 256C (2013)

[27] L. Kumar (for the STAR Collaboration), Nucl. Phys. A 931, 1114 (2014)

[28] L. Adamczyk et al. (STAR collabotration), Phys. Rev. C 93, 21903 (2016)

[29] B.I. Abelev et al. (STAR Collaboration), Phys. Rev. C 79, 064903 (2009)

[30] L. Adamczyk et al. (STAR collabotration), Phys. Rev. C 93, 014907 (2016)

[31] N. Yu (for the STAR Collaboration), "Beam energy dependence of $\mathrm{d}$ and anti-d production in $\mathrm{Au}+\mathrm{Au}$ collisions at RHIC", QM2015, September 27 - October 3, 2015 Kobe Fashion Mart, Kobe, Japan. http://qm2015.riken.jp/

[32] L. Adamczyk et al. (STAR collabotration), nucl-ex:1601.07052

[33] J. Cleymans et al., Phys. Rev. C 73, 034905 (2006). S. Wheaton, J.Cleymans, Comput. Phys. Commun. 180, 84 (2009)

[34] A. Andronic et al., Nucl. Phys. A 772, 167 (2006). A. Andronic et al., Phys. Lett. B 675, 312 (2009). A. Andronic et al., Nucl. Phys. A 834, 237C (2010)

[35] F. Becattini et al., Phys. Rev. C 73, 044905 (2006)

[36] E. Schnedermann, J. Sollfrank, U.W. Heinz, Phys. Rev. C 48, 2462 (1993)

[37] B.I. Abelev et al. (STAR Collaboration), Phys. Rev. C 79, 034909 (2009). B.I. Abelev et al. (STAR Collaboration), Phys. Rev. C 81, 024911 (2010)

[38] L. Kumar (for the STAR Collaboration), Nucl. Phys. A 904, 256c (2013)

[39] S. Das (for the STAR Collaboration), Nucl. Phys. A 904-905, 891c (2013)

[40] B. Huang (for the STAR Collaboration), "Beam Energy Scan Results from STAR", 247 (2015),

In: Proc. 50th Recontres de Moriond, "QCD and High Energy Interactions", La Thuile, Aosta Valley, Italy, March 21-28, 2015

[41] I. Zborovský, M. Tokarev, Phys. Rev. D 75, 094008 (2007)

[42] I. Zborovský, M. Tokarev, Int. J. Mod. Phys. A 24, 1417 (2009)

[43] M.V. Tokarev (for the STAR Collaboration), Int. J. Mod. Phys. Conf. Ser. 39, 1560103 (2015)

[44] STAR Collaboration, Technical Design Report: The STAR Heavy Flavor Tracker (2011)

[45] L. Ruan et al. (for the STAR Collaboration), J. Phys. G: Nucl. Part. Phys. 36, 095001 (2009)

[46] R. Ma (for the STAR Collaboration), "New STAR results", RHIC \& AGS Annual Users' Meeting, BNL, USA, January 7-10, 2016. https://www.bnl.gov/aum2016/ 
[47] M.Lomnitz (for the STAR Collaboration), "Open heavy flavor measurements in Au+Au collisions at $200 \mathrm{GeV}$ using the STAR Heavy Flavor Tracker", RHIC \& AGS Annual Users' Meeting, BNL, USA, January 7-10, 2016. https://www.bnl.gov/aum2016/

[48] H. Qiu (for the STAR Collaboration), "Heavy flavor measurements at the STAR experiment", 38th International Conference on High Energy Physics, Chicago, USA, August 3-10, 2016. http://www.ichep2016.org/

[49] M. Lomnitz (for the STAR Collaboration), "Review of recent heavy flavor measurements from the STAR experiment" 5th International Conference on New Frontiers in Physics (ICNFP 2016), Kolymbari, Greece, July 6-14, 2016. http://indico.cern.ch/event/442094/overview

[50] L. Adamczyk et al. (STAR Collaboration), Phys. Rev. Lett. 113, 142301 (2014)

[51] L. Adamczyk et al. (STAR Collaboration), Phys. Rev. D 86, 072013 (2012)

[52] Guannan Xie (for the STAR Collaboration), Quark Matter 2015, Kobe, Japan. Rongrong Ma (for the STAR Collaboration), 2016 RHIC \& AGS Annual Users Meeting, BNL, USA, June 7-10, 2015

[53] T. Todoroki (for the STAR collaboration) Strangeness in Quark Matter 2016, Berkeley, USA, June 27 - July 1, 2016. https://indico.cern.ch/event/403913/overview

[54] L. Adamczyk et al. (STAR Collaboration), Phys. Lett. B 722, 55 (2013)

[55] L. Adamczyk et al. (STAR Collaboration), Phys. Rev. C 90, 024906 (2014)

[56] D. Banerjee et al., Phys. Rev. D 85, 014510 (2012). H. Ding et al., J. Phys. G 38, 124070 (2010).

B.I. Abelev et al. (STAR collaboration), Phys. Rev. D 83, 052006 (2011)

[57] C. Alt et al. (NA49 Collaboration), Phys. Rev. C 77, 024903 (2008)

[58] D. Sebra (for the STAR Collaboration), "The Beam Energy Scans at RHIC: Results from BESI Prospects for BES-II", 34th Reimei Workshop "Physics of J-PARC Heavy-Ion Collisions", JPARC, Tokai, Japan, August 8-9, 2016. http://asrc.jaea.go.jp/soshiki/gr/hadron/workshop/reimei34 th/

[59] K.Meehan (for the STAR Collaboration), "The Fixed-Target Experiment at STAR", RHIC \& AGS Annual Users' Meeting, Upton, BNL, January 7-10, 2016. https://www.bnl.gov/aum2016/

[60] R.Reed (for the STAR Collaboration), "STAR Detector Upgrades" RHIC \& AGS Annual Users' Meeting, Upton, BNL, January 7-10, 2016. https://www.bnl.gov/aum2016/

[61] K.Meehan (for the STAR Collaboration), "The Fixed-Target Experiment at STAR", 32nd Winter Workshop on Nuclear Dynamics (WWND2016) 28 February - 5 March, 2016 , Guadeloupe, French. https://indico.cern.ch/event/435555/

[62] A. Schmah (for the STAR Collaboration), "STAR Upgrades and Physics Program", Strangeness in Quark Matter 2016, Berkeley, USA, June 27 - July 1, 2016. https://indico.cern.ch/event/403913/overview

[63] R. Reed (for the STAR Collaboration), "STAR Detector Upgrades", RHIC \& AGS Users' Meeting, 2016 RHIC \& AGS Annual Users' Meeting, Upton, BNL, January 7-10, 2016. https://www.bnl.gov/aum2016/

[64] STAR Collaboration, Proposal for STAR inner TPC sector Upgrade (iTPC). http://drupal.star.bnl.gov/STAR/starnotes/public/sn0619

[65] STAR Collaboration, "Technical Design Report for the iTPC Upgrade". December 1, 2015. https://drupal.star.bnl.gov/STAR/starnotes/public/sn0644

[66] C. Yang (for the STAR Collaboration), "The iTPCupgrade at STAR", Strangeness in Quark Matter 2016, Berkeley, USA, June 27 - July 1, 2016. https://indico.cern.ch/event/403913/overview [67] STAR Collaboration, eTOF Proposal, January, 2016 
[68] STAR Collaboration, An Event Plane Detector for STAR, Proposal, May, 2016. https://drupal.star.bnl.gov/STAR/system/files/

[69] W. Fischer, "RHIC upgrades for the next decade", Satellite Workshop "Accelerators Revealing the QCD Secrets", XIIth Quark Confinement and Hadron Spectrum, Thessaloniki, Greece, 4 September, 2016

[70] White Paper "Low-Energy RHIC electron Cooler (LEReC)", September 19, 2013

[71] A. Fedotov, "LEReC overview: project goal and cooling approach", Low Energy RHIC electron Cooling (LEReC), MEIC Collaboration Meeting, 30-31 March, 2015

[72] D. Sebra (for the STAR Collaboration), "Exploring the QCD Phase Diagram: RHIC Beam Energy Scan II", Low Energy RHIC electron Cooling Review, BNL, USA, 13 August, 2013

[73] H.E. Stanley, Introduction to Phase Transitions and Critical Phenomena (Oxford, New York and Oxford, 1971)

[74] H.E. Stanley, Rev. Mod. Phys. 71, S358 (1999)

[75] M. Tokarev, I. Zborovský, Nucl. Phys. Proc. Suppl. B 245, 231 (2013) 